\title{
O uso de Digitalização 3D em experiências de documentação digital de patrimônio histórico: o caso da Sede Social do Metropolitano de Lisboa
}

\author{
The use of 3D Digitization in experiences of digital documenting historical heritage: the case of Lisbon's Metropolitano Social \\ Headquarters \\ - Leticia Teixeira Mendes \\ Universidade Federal de Pernambuco, Brasil \\ Maycon Sedrez \\ Universidade Estadual de Campinas, Brasil \\ leticia.mendes@ufpe.br \\ mayconsedrez@gmail.com \\ - Cristiana Griz \\ Universidade de Pernambuco, Brasil \\ crisgriz@gmail.com
}

\begin{abstract}
This paper describes the use of $3 \mathrm{D}$ digitization to acquire data and to produce technical documentation for the inventory of historical buildings. The object defined as case study is an ornamented window of the Lisbon's Metropolitano Social Headquarters, project of the architect Norte Junior, located in the city of Lisbon. The methodology of the research relies on the comparison of two different approaches: (1) the use of Autodesk 123D Catch software to produce a geometric model and (2) the of the software My 3D Scanner to obtain a point cloud and the MeshLab software to obtain a texturized mesh.
\end{abstract}

Keywords: 3D Digitization, Architectural Heritage, Photogrammetry, Documentation Technologies, Preservation

\section{Introdução}

A utilização de novas tecnologiaspara olevantamento dedados e elaboração de documentos técnicos que complementem os inventários de patrimônio histórico mostra-se extremamente eficazes em um campo onde o detalhamento e informações existentes são escassos e de difícil acesso. Segundo Celani e Cancherini (2009), a utilização de fotografias para a obtenção de dados tridimensionais pelo método da perspectiva inversa é uma técnica muito antiga, que permite extrair dimensões a partir de fotografias de objetos dos quais ao menos algumas dimensões e ângulos são conhecidos. Os métodos fotogramétricos tridimensionais pressupõem sempre a existência de duas ou mais imagens, obtidas por meio de pontos de vista diferentes e com sobreposição, ou seja, os elementos presentes na cena devem ser representados em pelo menos duas imagens (Mateus, 2012). Com a disponibilidade de equipamentos óticos digitais de alta precisão e de técnicas de visão computacional, essa área tomou grande impulso, dando origem ao que hoje se chama digitalização tridimensional.

Para obtenção dos objetivos do experimento descrito neste artigo - a digitalização de um objeto de pequena ou média escala - serão utilizadas técnicas fotogramétricas. Segundo Mikhail et al. (2001) a fotogrametria é considerada ciência e arte, cujo objetivo é estudar os processos pelos quais se pode derivar informação, em geral métrica, sobre um objeto através de medições efetuadas em fotografias do objeto. A tarefa fundamental da fotogrametria é estabelecer de forma rigorosa a relação geométrica entre a fotografia e o objeto no momento em que esta foi obtida.

Atualmente, os procedimentos digitais são recorrentes na documentação e levantamento histórico devido à sua praticidade e baixo custo. Esta técnica, utilizada no presente exercício, otimiza a obtenção de dados métricos de objetos, edifícios, ornamentos, entre outros; minimizando assim, a etapa das medições tradicionais (Oliveira, 2008). Dessa forma, buscou-se a utilização de múltiplas imagens planas que, devidamente orientadas, permitem a construção de modelos 3D e a restituição gráfica dos objetos representados; possibilitando assim, a utilização do modelo para extrair áreas, comprimentos e volumes com distribuições espaciais. Ainda é possível utilizar esses modelos para visualização em ambientes virtuais ou como elementos integrantes de bases de dados (Mateus, 2012).

\section{Objetivos}

Este trabalho objetiva apresentar as etapas de produção de um modelo tridimensional fechado e texturizado de um objeto de média escala utilizando técnicas de fotogrametria. Para isso, esta pesquisa objetivou comparar o uso de dois métodos distintos para a coleta de dados de um edifício histórico, em particular, a digitalização 3D de uma janela. Os instrumentos utilizados foram: (1) software Autodesk 123D Catch, que utiliza processamento em nuvem, para produzir 
um modelo geométrico final, e (2) a geração do mesmo objeto através de um processo mais complexo, a partir do uso do software My 3D Scanner para obter a nuvem de pontos e o software MeshLab para gerar a malha texturizada.

\section{Instrumentos Metodológicos}

\section{Fotogrametria para reconstrução 3D}

Anterior à escolha do objeto, foram executados diversos testes com diferentes objetos e texturas. As simulações que precederam a escolha do objeto para o exercício foram fundamentais para analisar os resultados obtidos nos testes de acordo com a variação de material, luminosidade e escala de cada objeto. A escolha do objeto para o desenvolvimento deste experimento baseou-se nas características que resultaram melhor qualidade da nuvem de pontos nos testes anteriores - textura opaca, superfície rugosa, riqueza em detalhes e ornamentos - contribuindo assim, para avaliar as tecnologias testadas e maior chance de obter resultados satisfatórios.

\section{Aquisição das imagens}

A aquisição das imagens ocorreu em dias nublados para obter melhores resultados devido a menor interferência de luz e reconhecimento das imagens na geração da nuvem de pontos. O objeto escolhido contribuiu para a análise das tecnologias, pois apresenta superfície opaca, rugosidade e embora seja simétrico, possui riqueza de detalhes. As fotografias devem ser capturadas a partir de diferentes ângulos, espacialmente dispostas em um círculo ou arco em torno do objeto e cada foto deve conter cerca de $70-80 \%$ do conteúdo da imagem capturada anteriormente.

\section{Obtenço da nuvem de pontos}

As ferramentas comparadas para geração da nuvem de pontos da janela do edifício histórico foram: Autodesk 123D Catch e My 3D Scanner. A primeira ferramenta é um software autônomo instalado no computador do usuário, cujo serviço gratuito oferecido pela Autodesk, permite processar automaticamente até 70 fotos. Os dados (coleção de fotos) são transmitidos ao banco de dados da Autodesk para processar a informação, após esse processo o usuário tem acesso a um arquivo de dados das fotos da cena capturada (Photo Scene Data File). O usuário pode editar e enviar esse arquivo para outros programas em diversas extensões, como *.dwg, ${ }^{*}$.fbx, ${ }^{*}$.obj, ${ }^{*}$.las. O resultado final consiste no processo de reconstrução contendo a nuvem de pontos, a malha 3D (mesh) e a textura.

A ferramenta My 3D Scanner é um serviço online gratuito que permite processamento de até 100 imagens para gerar uma nuvem de pontos. $\mathrm{O}$ produto final disponibilizado pelo aplicativo consiste em um arquivo de malha 3D (extensão *obj) e um arquivo de nuvem de pontos (extensão *.ply).

\section{Estudo de Caso}

O objeto escolhido para digitalização foi uma das janelas (fig. 1) do edifício da Sede Social do Metropolitano de Lisboa, projetado pelo Arquiteto Norte Júnior, localizado na interseção da Avenida Fontes Pereira de Melo e Rua Andrade Corvo, no bairro de Picoas, na cidade de Lisboa. $O$ edifício, popularmente conhecido por bolo de noiva, devido a exuberante ornamentação da fachada, foi vendido à Câmara Municipal de Lisboa em 1950, posteriormente alugado para o Metropolitano de Lisboa em 1954, onde se instala atualmente a sede administrativa da empresa de transportes.

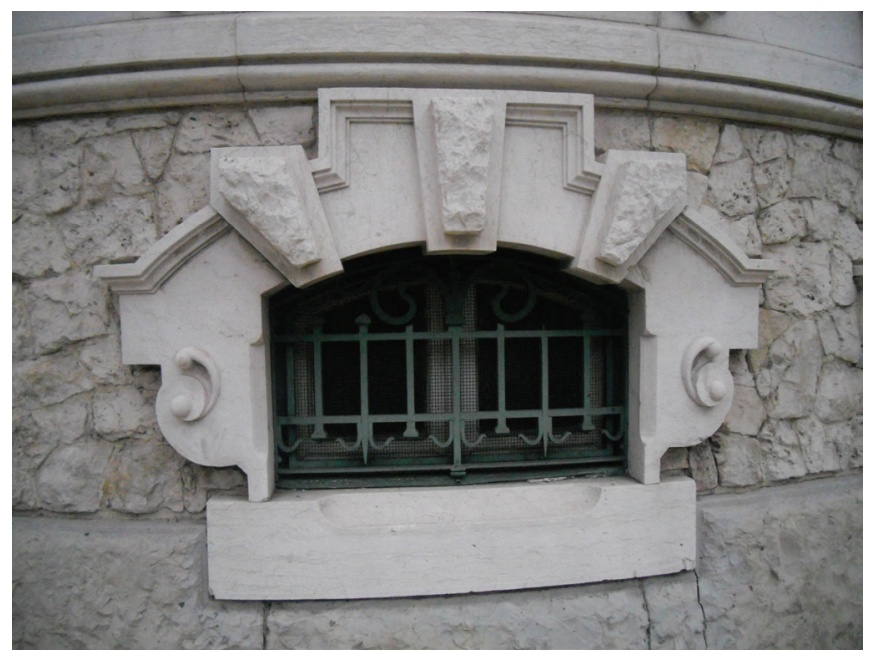

Figura 1: Janela selecionada do Metropolitano de Lisboa. Dos autores, 2014.

Conforme descrito anteriormente, as etapas para reconstruir as superfícies exteriores de uma das janelas do edifício histórico se basearam no uso dos seguintes programas: (1) Autodesk 123D Catch e (2) My 3D Scanner. Para isso, o primeiro passo constituiu-se na captura das fotografias do objeto a partir de ângulos diferentes (fig. 2) para possibilitar a aplicação dos algoritmos de reconstrução por meio dos programas escolhidos.

\section{Teste ${ }^{\circ}$ 1: Autodesk 123D Catch}

As etapas para gerar um modelo 3D no Autodesk 123D Catch são constituídas pelos seguintes passos: criar um novo projeto e fazer o upload das fotos, esse processo pode demorar vários minutos, dependendo da quantidade de imagens e da complexidade do objeto. O número de fotos depende do objeto que será digitalizado, neste experimento, digitalização da janela, foram utilizadas 68 fotografias. Após o processamento do modelo 3D, este pode ser editado através da adição de mais fotos, ajustando fotos que foram ignoradas pelo servidor ou remover partes desnecessárias, como mostra a fig. 2 em vermelho. Também é possível visualizar o mesh criado pelo programa (fig. 3).

Neste teste foi utilizado a versão online da ferramenta Autodesk 123D Catch. Existem algumas diferenças entre a versão online e a versão autônoma, que possui recursos extras como a definição do sistema de coordenadas geográficas 


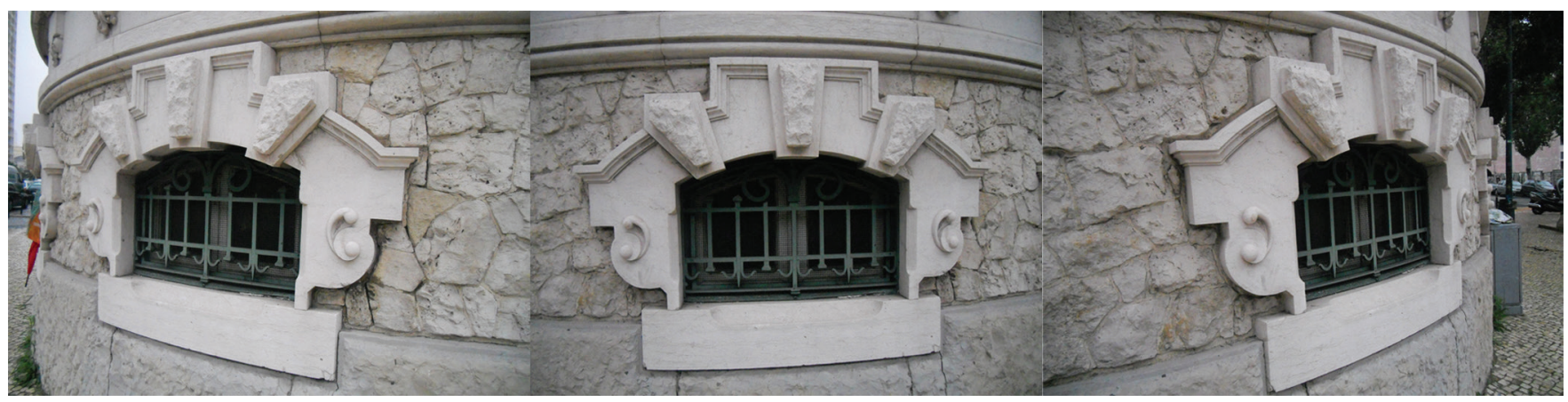

Figura 2: Fotografias de diferentes ângulos. Dos autores, 2014.

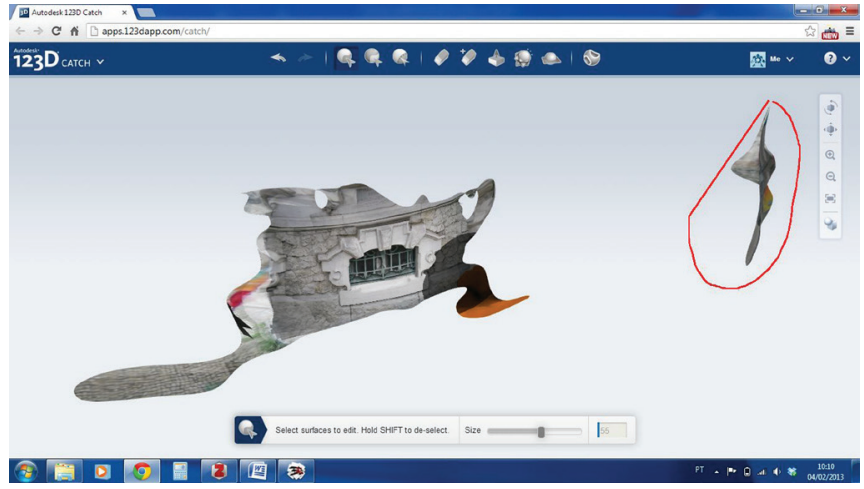

Figura 3: Edição da janela digitalizada no 123D Catch . Dos autores, 2014.

mundial, qualidade da malha (mesh), criação de pontos de referência ou linhas e visualização do percurso das fotos que compõem o modelo. No entanto, a versão online também possui os recursos necessários para edição das superfícies lisas, criação de um plano de corte e inspeção do modelo 3D (para fechar os buracos no objeto permitindo sua impressão em 3D).

\section{Teste $\mathrm{n}^{\circ}$ 2: My 3D Scanner + MeshLab}

Nesta seção são apresentados os passos para geração de um modelo 3D a partir do uso da ferramenta online My 3D Scanner para obtenção de uma nuvem de pontos (fig. 4) e definição de uma malha (mesh) texturizada por meio do software MeshLab.

O processo foi constituído pelos seguintes passos: (1) fazer o upload das 68 fotos da janela para o site do My 3D Scanner,

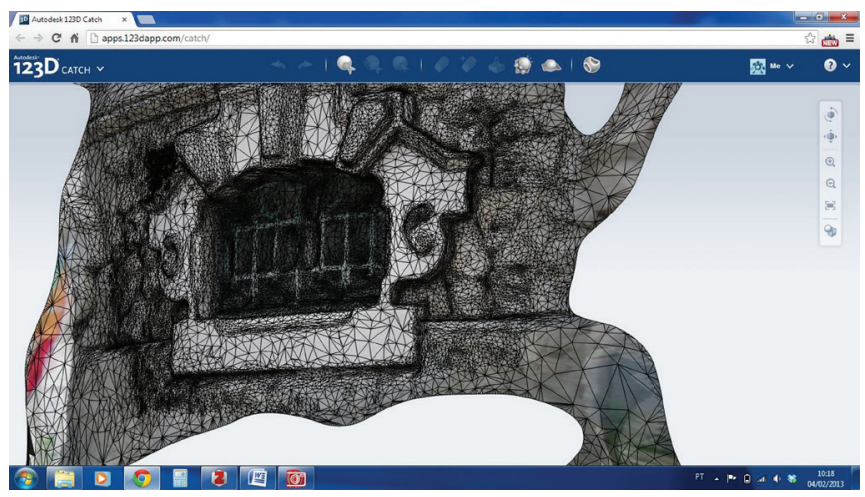

Figura 4: Janela digitalizada com textura e mesh no 123D Catch . Dos autores, 2014.

depois de algumas horas o usuário recebe uma mensagem por correio eletrônico informando cada etapa do processo. A possibilidade de utilizar o processamento das imagens por meio do serviço online otimiza o tempo para execução da tarefa sem interferir no desempenho do computador do usuário. (2) Abrir o arquivo .obj (resultado da etapa anterior) no software MeshLab para edição da nuvem de pontos, removendo pontos desnecessários e limpando resíduos no entorno do objeto digitalizado. O software Meshlab foi usado para reconstrução da superfície 3D a partir da nuvem de pontos geradas por meio da ferramenta My 3D Scanner, dessa forma, os pontos que não faziam parte do objeto 3D reconstruído foram excluídos usando os comandos: Selecionar Vértices (para selecionar os pontos) e Excluir atual conjunto de vértices selecionados (para excluir os pontos). (3) A última etapa consistiu na geração da malha (mesh) e texturização (fig. 5). 


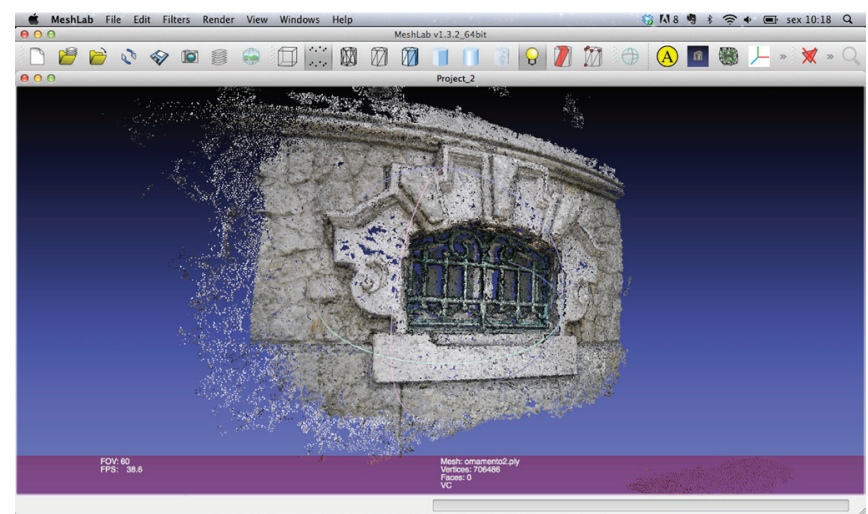

Figura 5: Nuvem de pontos no My 3D Scanner. Dos autores, 2014.

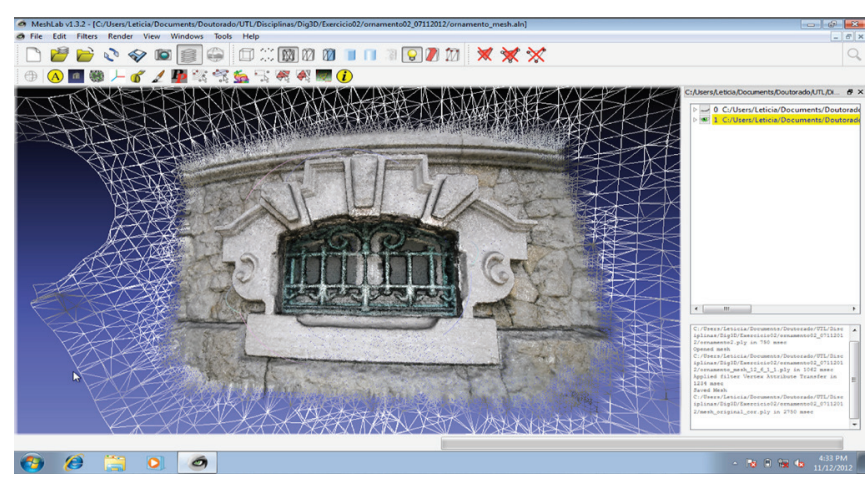

Figura 6. Malha gerada com textura no MeshLab. Dos autores, 2014.

\section{Discussão}

Conforme o experimento descrito acima, é possível dizer que os dois métodos produziram resultados semelhantes, com a única diferença de que, no primeiro método, apenas um programa foi utilizado e, portanto, todo o processo foi mais rápido. A versão online do software My 3D Scanner não está mais disponível para acesso. Dessa forma, pretende-se testar alternativas open source como o visualSFM e o openMVG, ambos disponíveis para serem instalados no computador do usuário.

As técnicas de digitalização 3D tendem a ser adotadas na documentação de patrimônio histórico de elementos ornamentais, por exemplo, permitindo sua reprodução com tecnologias como a impressão 3D ou métodos artesanais. Algumas das vantagens do uso de programas gratuitos de digitalização 3D para o ensino de arquitetura são: inclusão de novas tecnologias no estudo de patrimônio histórico, replicação das técnicas de fotogrametria para outras áreas da arquitetura como o projeto (método de projeto adotado por
Frank Ghery no projeto do Museu Guggenheim de Bilbao (Ferrari, 2011)); familiarizando o aluno com as possibilidades tecnológicas existentes.

A contribuição deste artigo é a disseminação da experimentação com processos de digitalização para a documentação de patrimônio histórico. Outros testes precisam ser realizados com o objetivo de comparar os métodos em termos de precisão dimensional e de geração de arquivos com extensão *.STL para a produção de modelos com o uso de tecnologias de prototipagem rápida. A fim de produzir um modelo adequado para impressão 3D a malha precisa ser contínua, sem quaisquer imperfeições. $O$ processo de produção de modelos físicos também pode contribuir para a compreensão e documentação de edifícios históricos.

\section{Agradecimentos}

O presente trabalho foi realizado com o apoio do CNPq, Conselho Nacional de Desenvolvimento Científico e Tecnológico - Brasil e da Fundação de Amparo à Pesquisa do Estado de São Paulo - FAPESP (Processos no 2012/10498-3, 2011/50139-0, 2013/09362-2 e 2014/13572-5).

\section{Referências}

Celani, G., Cancherini, L. (2009). Digitalização tridimensional de objetos: um estudo de caso. Anais do Sigradi 2009. São Paulo, p. 309-311.

Ferrari, D. O. A. (2011). Estudo comparativo entre processo criativo na arquitetura e na joalheria com ênfase nas criações de Frank Gehry. Dissertação de Mestrado. Universidade de São Paulo. São Paulo.

Heuvel, F. V. D. (2001). Reconstruction from a single architectural image from the Meydenbauer archives. Proceedings do XVIII CIPA Symposium 2001. Postdam.

Oliveira, M. M. (2008). A documentação como ferramenta de preservação da memória. Brasília, DF: IPHAN / Programa Monumenta, 144 p. (Cadernos Técnicos ; 7).

Mateus, L. M. C. (2012). Contributos para o projecto de conservação, restauro e reabilitação - Uma metodologia documental baseada na fotogrametria digital e no varrimento laser 3D terrestres. Tese de Doutoramento. Faculdade de Arquitectura. Universidade Técnica de Lisboa.

Mikhail, E., Bethel, J., McGlone, J. (2001). Introduction to modern photogrammetry. EUA: John Willey \& Sons.

Pavlidis, G., Koutsoudis, A., Arnaoutoglou, F., Tsioukas, V., Chamzas, C. (2007). Methods for 3D digitization of Cultural Heritage, Journal of Cultural Heritage, 8, p. 93-98. 